\title{
The efficacy of oral anticholinergics for sympathetic overactivity in a thoracic surgery clinic
}

\author{
Conor F. Hynes, MD, Sulakshana Seevaratnam, BS, Krisana Gesuwan, NP, Marc Margolis, MD, and \\ M. Blair Marshall, MD
}

\section{ABSTRACT}

Objective: Little is known of the success rates of oral anticholinergics for the treatment of primary hyperhidrosis and facial blushing as alternatives to surgical intervention. We examine predictors of success with these medications.

Methods: A retrospective review was performed at a single institution, including all patients presenting with symptoms of primary hyperhidrosis, facial blushing, or both from 2004 to 2015 . All patients were offered a trial of oral anticholinergics. If oral anticholinergic therapy was not successful, patients were offered surgery. Statistical analyses were performed to compare patients who declined surgery given the trial of oral anticholinergics with those who proceeded with surgery.

Results: A total of 381 patients presented with symptoms of primary hyperhidrosis $(86.6 \%)$, facial blushing $(2.4 \%)$, or both $(11.0 \%)$. A total of 230 patients $(60.4 \%)$ declined surgery after using oral anticholinergics, and 151 patients $(39.6 \%)$ chose surgery. Patients who declined surgery were more likely to have symptoms of primary hyperhidrosis without facial blushing $(89.6 \%$ vs $82.1 \%$; $P=.02$ ) or have primary symptoms involving the axilla, torso, scalp, or groin. Patients who proceeded with surgery had higher rates of palmar symptoms as a primary site $(77.6 \%$ vs $61.1 \% ; P=.01)$ and were more likely to have facial blushing alone or in combination with primary hyperhidrosis. Presentation with palmar symptoms and greater number of prior therapy attempts were independent predictors of proceeding with surgery after controlling for concomitant symptom type and location $(P=.01$ and $P<.0001$, respectively).

Conclusions: The majority of patients presenting with sympathetic overactivity decline surgery when a trial of oral anticholinergics is included in the treatment algorithm. Facial blushing and palmar symptoms were each associated with choosing surgery. (J Thorac Cardiovasc Surg 2016;152:565-8)

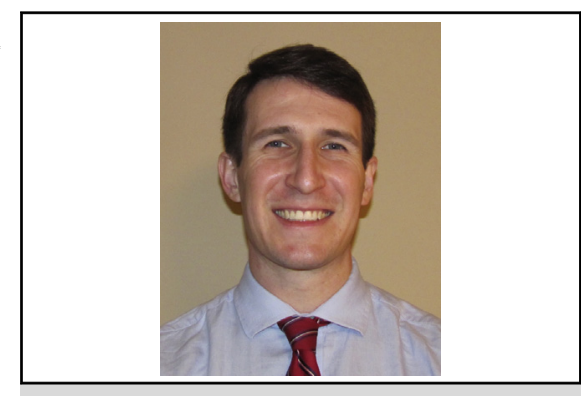

Conor F. Hynes, MD

Central Message

Some $60 \%$ of patients with sympathetic overactivity are well managed with OAs with no risk of compensatory sweating.

\section{Perspective}

Sympathetic overactivity is a devastating condition that is effectively treated surgically. However, surgical therapies portend a notable risk of compensatory sweating, which can be equally debilitating. OA therapies can offer sufficient symptom management in a majority of patients, and several factors can predict their success.

See Editorial Commentary page 569.
Primary hyperhidrosis (PH) and facial blushing (FB) represent 2 forms of sympathetic overactivity that often present together and are managed similarly. For both conditions, the most effective treatment is surgical interruption of the sympathetic chain at the rib level corresponding to symptoms, via the procedure known as "endoscopic thoracic sympathectomy" (ETS). Because of the favorable efficacy,

\footnotetext{
From the Division of Thoracic Surgery, Georgetown University Hospital, Washington, DC.

Received for publication Jan 3, 2016; revisions received March 3, 2016; accepted for publication March 13, 2016; available ahead of print May 6, 2016.

Address for reprints: M. Blair Marshall, MD, Division of Thoracic Surgery, George-

town University Hospital, 3800 Reservoir Rd, NW, Washington, DC 20007

(E-mail: conor.f.hynes@gmail.com).

$0022-5223 / \$ 36.00$

Copyright (c) 2016 by The American Association for Thoracic Surgery

http://dx.doi.org/10.1016/j.jtcvs.2016.03.092
}

straightforward approach, and tolerance by patients, ETS has become a mainstay of therapy. The important downfall of this procedure is the relatively high rate of compensatory sweating, which is reported in some degree by the majority of patients and leads to requests for reversal in approximately $10 \%$ of ETS recipients. ${ }^{1}$

There is a wide selection of nonsurgical therapies in use that avoid the issue of compensatory sweating and therefore are recommended as first-line treatment. However, these are

Scanning this $\mathrm{QR}$ code will take you to the article title page.

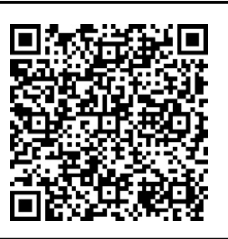




\section{Abbreviations and Acronyms}

ETS $=$ endoscopic thoracic sympathectomy

$\mathrm{FB}=$ facial blushing

$\mathrm{OA}=$ oral anticholinergic

$\mathrm{PH}=$ primary hyperhidrosis

less effective in severe cases, and many of these modalities have undergone limited scientific evaluation from which to draw conclusions. Oral anticholinergic (OA) medications, specifically glycopyrrolate and oxybutynin, are supported by a body of literature that continues to expand $d^{2-5}$ and, given their systemic nature, offer the benefit of treating multifocal disease, a common presentation in this patient population. Many patients have symptoms in a combination of sites, which can include the face, palms, axillae, trunk, groin, and feet. Combinations can be cumbersome and often frustrating to treat with local therapies that require recurrent and multifocal administrations. Application of ETS also is limited in this regard, because it has no ability to address symptoms below the thorax and carries a risk of injuring the stellate ganglion superiorly. Surgeons have developed over time a sharper understanding of how to select patients for surgery, which requires discussion of patient expectations in addition to evaluation of symptom site and severity. We report our experience with OA medications as first-line therapy and analyze factors associated with the preference of OAs over surgery.

\section{PATIENTS AND METHODS}

A retrospective review of patients referred to a thoracic surgery clinic for the diagnosis of sympathetic overactivity at a single institution was performed after Institutional Review Board approval. All patients presenting from November 2004 to February 2015 were reviewed. Some $99 \%$ had symptoms of primary sympathetic overactivity meeting inclusion criteria, whereas $1 \%$ presented with hyperhidrosis secondary to other causes and therefore were excluded. Patients were initially offered oral glycopyrrolate therapy at a dose of $1 \mathrm{mg}$ twice per day and titrated up as needed according to symptoms to a maximum of $8 \mathrm{mg}$ twice per day. If symptoms were not adequately controlled or adverse effects (primarily xerostomia) were intolerable, a switch to oxybutynin was then offered at 5 to $10 \mathrm{mg}$ per day and titrated up to a maximum of $30 \mathrm{mg}$ per day as needed. If neither medication adequately controlled symptoms, the risks and benefits of ETS were discussed and the procedure was offered to patients who believed the benefits outweighed the risks (Figure 1). Patients who previously underwent ETS were excluded.

The primary outcome evaluated was the proportion of patients who declined surgery after being offered OA medication. Patients managed with OA medications who declined surgery and continued with nonoperative therapy were compared with patients who proceeded with ETS despite oral therapy. Univariate analyses of patient characteristics, diagnosis types, symptom sites, and prior therapies were performed using the $t$ test for continuous variables and the chi-square test for proportions.

Multivariable logistic regression analysis was performed to evaluate predictors of declining surgery while controlling for age, sex, diagnosis type, symptom location, and prior therapies. Statistical analysis was performed using IBM SPSS Statistics (version 21.0, IBM, Armonk, NY).
Patients referred to thoracic surgery clinic for primary hyperhidrosis

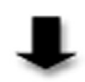

Offered glycopyrrolate $1 \mathrm{mg}$ twice per day:

If inadequate relief without intolerable side effects

$\rightarrow$ titrated up to maximum $8 \mathrm{mg}$ twice per day

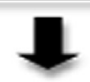

If inadequate symptom relief or perceived futility
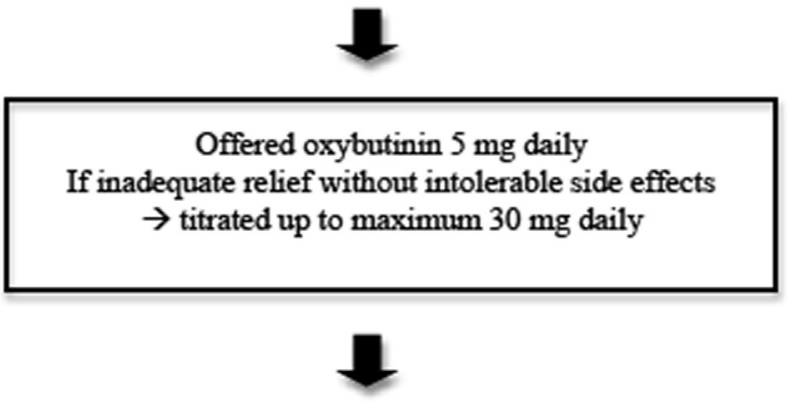

Inadequate symptom relief or intolerable side effects

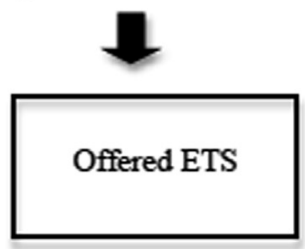

FIGURE 1. PH treatment algorithm. ETS, Endoscopic thoracic sympathectomy.

\section{RESULTS}

During the 10-year period, 381 patients presented with symptoms of primary sympathetic overactivity and were offered our standard treatment algorithm. The majority were patients referred by another provider from the District of Columbia, Northern Virginia, and Western Maryland. Of these, $330(86.6 \%)$ were diagnosed with PH, $9(2.4 \%)$ were diagnosed with isolated $\mathrm{FB}$, and $42(11.0 \%)$ were diagnosed with a combination of both. A total of 230 patients $(60.4 \%)$ persisted with nonoperative management and 151 patients $(39.6 \%)$ proceeded with surgery. For the surgery group, the median time from initial presentation to surgery was 71 days (interquartile range, 36-202 days). Mean follow-up for the nonoperative group was 304 days (range, 0 days to 6.8 years).

A total of 293 patients had received some form of prior therapy, which was prior OAs in 65 of these. Other previous therapy modalities included iontophoresis, aluminum 
chloride antiperspirants, and botulinum toxin injections. A total of 314 patients $(82 \%)$ proceeded with a trial of OA medications after visiting our clinic. Of the 67 patients who did not accept any OA therapy after visiting our clinic, 60 had attempted some form of prior therapy and 29 had specifically attempted OAs previously and did not want to try again. Fifty-one of the patients refusing further OA therapy elected to proceed directly with ETS at that time.

Patients persisting with nonoperative management were significantly more likely to present with $\mathrm{PH}$ symptoms without FB compared with those who proceeded with surgery $(89.6 \%$ vs $82.1 \% ; P=.02)$ and were more likely to have symptoms in the scalp $(10.4 \%$ vs $4.6 \% ; P=.04)$, axillae $(44.8 \%$ vs $31.8 \% ; P=.009)$, torso $(10.9 \%$ vs $0.7 \% ; P<.0001)$, and groin $(5.7 \%$ vs $0.7 \% ; P=.01)$ (Table 1). They were also significantly less likely to have previously tried glycopyrrolate or iontophoresis. The median dose of glycopyrrolate used in the nonoperative group to control symptoms was $1 \mathrm{mg}$ twice per day, with a maximum of $8 \mathrm{mg}$ twice per day. Ten of these patients switched to oxybutynin.

Patients who proceeded with surgery had higher rates of isolated FB $(4.0 \%$ vs $1.3 \% ; P=.09)$ or a combination of FB and PH (14.6\% vs $8.7 \% ; P=.07)$ compared with the nonoperative group, although the differences did not reach statistical significance. Patients in the surgery group were significantly more likely to have palmar $\mathrm{PH}$ symptoms $(78.1 \%$ vs $59.1 \% ; P<.0001)$ and to have tried a greater number of prior therapies $(1.53 \pm 1.1$ vs $1.09 \pm 1.0$; $P=.005)$ than the nonoperative group. The median dose of glycopyrrolate used in the group that ultimately proceeded with surgery was $1.5 \mathrm{mg}$ twice per day. Five of these patients had switched to oxybutynin before choosing surgery.

Logistic regression analysis was performed to evaluate predictors of persistence with nonoperative therapy. Binary variables included in the model were sex, symptom site, and class of symptoms (ie, PH alone vs FB), in addition to the continuous variables age and number of prior therapies. The symptom presentation of combined PH and FB symptoms was withheld from the regression model because of multicollinearity with the presentations of $\mathrm{PH}$ or $\mathrm{FB}$ alone; however, none of the remaining variables were problematic. No positive predictors of persistence with nonoperative therapy were identified. When controlling for the aforementioned variables, the model showed that the presence of palmar symptoms and a history of a greater number of previously attempted therapies were each negative predictors of persistence with nonoperative management $(P<.0001$ for each).

\section{DISCUSSION}

To optimize outcomes for patients with sympathetic overactivity, identification of patients who can be
TABLE 1. Nonoperative versus operative

\begin{tabular}{|c|c|c|c|}
\hline & $\begin{array}{c}\text { Nonoperative } \\
\quad(\mathbf{n}=\mathbf{2 3 0})\end{array}$ & $\begin{array}{c}\text { Operative } \\
(n=151)\end{array}$ & $P$ value \\
\hline Age, y & $30.0 \pm 11.7$ & $29.5 \pm 11.5$ & .68 \\
\hline Male & $93(40.4 \%)$ & $62(41.1 \%)$ & .97 \\
\hline HH only & $206(89.6 \%)$ & $124(82.1 \%)$ & .02 \\
\hline Isolated FB & $3(1.3 \%)$ & $6(4.0 \%)$ & .09 \\
\hline Combined $\mathrm{HH}$ and FB & $20(8.7 \%)$ & $22(14.6 \%)$ & .07 \\
\hline Primary facial symptoms & $52(22.6 \%)$ & $38(25.2 \%)$ & .61 \\
\hline Primary palmar symptoms & $136(59.1 \%)$ & $118(78.1 \%)$ & $<.0001$ \\
\hline Primary axillary symptoms & $103(44.8 \%)$ & $48(31.8 \%)$ & .009 \\
\hline Primary torso symptoms & $25(10.9 \%)$ & $1(0.7 \%)$ & $<.0001$ \\
\hline Primary inguinal symptoms & $13(5.7 \%)$ & $1(0.7 \%)$ & .01 \\
\hline Primary pedal symptoms & $115(50.0 \%)$ & $85(56.3 \%)$ & .28 \\
\hline Primary scalp symptoms & $24(10.4 \%)$ & $7(4.6 \%)$ & .04 \\
\hline No. of attempted treatment modalities & $1.09 \pm 1.0$ & $1.53 \pm 1.1$ & .005 \\
\hline Prior iontophoresis & $26(11.3 \%)$ & $37(24.5 \%)$ & .001 \\
\hline Prior topical aluminum chloride & $141(61.3 \%)$ & $105(69.5 \%)$ & .13 \\
\hline Prior glycopyrrolate & $19(8.3 \%)$ & $44(29.1 \%)$ & ) $<.0001$ \\
\hline Prior oxybutynin & $4(1.7 \%)$ & $3(2.0 \%)$ & .87 \\
\hline Prior botulinum toxin & $19(8.3 \%)$ & $18(11.9 \%)$ & .25 \\
\hline
\end{tabular}

Continuous variables expressed as mean \pm standard deviation. Proportions expressed as percentages. Boldface indicates statistical significance. $H H$, Hyperh idrosis; $F B$, facial blushing.

successfully managed nonoperatively is crucial. Although direct comparison of ETS and nonoperative therapies presents a trial design challenge, higher success rates suggest ETS is most effective for severe PH and FB. However, the adverse effect of compensatory sweating has been highlighted in the literature as a cause for concern because of considerable numbers of ETS recipients expressing regret and requesting reversal. Many nonoperative management options have been explored in response to this treatment dilemma, although each has its own limitations.

Topical medications offer relief for milder symptoms with minimal side effects. Most notably, botulinum toxin has proved an effective albeit temporary and costly therapy. Many ingenious local device therapies also have been tested, including fractional radiofrequency, iontophoresis, lasers, and ultrasound but have established mixed results. ${ }^{6}$ The most limiting aspects of topical and localized therapies are their transient efficacy and the challenge to treating multifocal disease.

Systemic therapy thus has emerged as an effective alternative that also provides a solution to the issue of multifocality, with a dose-dependent and self-limited side effect profile. OAs are reported in the literature to provide improvement of $\mathrm{PH}$ symptoms in $67 \%$ to $80 \%$ of patients, with some variation by symptom site. Treatment failure due to lack of efficacy or the major adverse effect, xerostomia, has been reported in $25 \%$ to $33 \%$ of cases. ${ }^{3,4,7}$ Our results support these findings and further indicate that the symptom site predicts success with nonoperative therapy. 
Several authors have previously noted enhanced success rates in specific body sites. ${ }^{4,8}$ One randomized placebocontrolled trial found oxybutynin to improve pedal symptoms in $90 \%$ of cases. ${ }^{1} \mathrm{PH}$ presenting in regions outside the dermatomes of the thoracic sympathetic chain is the most logical beneficiary of systemic therapy and was successfully treated with OAs for scalp, torso, and groin symptoms. The lone exception was pedal symptoms, which did not differ between groups. It is counterintuitive that pedal symptoms would receive a greater benefit from ETS, given that their sympathetic innervation is extrathoracic; however, pedal symptoms often present with other sites, which actually may be the drivers of desire for surgery.

Palmar PH and FB were the 2 presentations more highly associated with proceeding with surgery. Because these are the most socially problematic sites, it is likely that patients have a lower tolerance for even mild symptoms in these areas. Functional impediments also are greater at these sites. Palmar symptoms limit work in a wide range of vocations, for example, office settings that require frequent use of paper, pen, and handshakes. FB presents its own functional limitations in terms of emotional interaction, social avoidance, and communication. By contrast, axillary symptoms were more often successfully treated with OAs for our patients, potentially because hyperhidrosis at that site is more socially excusable and creates no functional limitations.

An important consideration is that treatment of $\mathrm{FB}$ is distinct from PH. Studies of systemic therapy for FB are mostly observational and indeterminate. ${ }^{9}$ Furthermore, the pathologic phenomenon of FB is the anxiety surrounding the blushing rather than the actual occurrence, and it is unclear whether those with FB have greater blood flow or even frequency of blushing compared with the general population. ${ }^{10}$ Accordingly, nonoperative treatment focuses on patient anxiety with the use of anxiolytics, beta-blockers, and cognitive behavioral therapy. ${ }^{9}$ Despite the differences in pathophysiology and nonoperative treatment paradigms, ETS has been found to be as effective for isolated FB as for $\mathrm{PH},{ }^{11}$ if not better, with rates of improvement of approximately $90 \% .^{12,13}$ When compared with anxiolytic therapy, ETS produced $30 \%$ greater satisfaction, ${ }^{12}$ and rate of regret is similar at $10 \% .{ }^{13}$ The results of our study suggest OAs are less effective for FB, and these patients might benefit from earlier operative therapy.

\section{Study Limitations}

This study is limited predominately by the retrospective design. The level of satisfaction was not objectively stratified, and therefore we relied on treatment decisions. Although it would be useful to characterize the degree of satisfaction in the OA group, it is relevant to distinguish patients who opt for surgery from those who prefer nonoperative management in light of individual perceptions of symptoms and risk of therapy. The lack of complete understanding by the medical community regarding the true mechanism of ETS and its success further complicates the decision-making process. Finally, the number of cases of FB was low, limiting statistical power; however, this is representative of the overall prevalence.

\section{CONCLUSIONS}

Our results show that OA therapy is effective in many patients with sympathetic overactivity and that nonoperative treatment failure can be predicted by symptom sites that are the most socially and functionally limiting. Because of the relevant rates of regret after ETS, surgical intervention for symptoms at other sites requires first an earnest effort to exhaust nonoperative therapies and a thorough discussion with patients regarding their perceptions of the risks and benefits of an operation.

\section{Conflict of Interest Statement}

Authors have nothing to disclose with regard to commercial support.

\section{References}

1. Hynes CF, Yamaguchi S, Bond CD, Marshall MB. Reversal of sympathetic interruption by removal of clips. Ann Thorac Surg. 2015;99:1020-4.

2. Wolosker N, de Campos JR, Kauffman P, Puech-Leao P. A randomized placebocontrolled trial of oxybutynin for the initial treatment of palmar and axillary hyperhidrosis. J Vasc Surg. 2012;55:1696-700.

3. Benson RA, Palin R, Holt PJ, Loftus IM. Diagnosis and management of hyperhidrosis. BMJ. 2013;347:f6800.

4. Teivelis MP, Wolosker N, Krutman M, Kauffman P, de Campos JR, PuechLeao P. Treatment of uncommon sites of focal primary hyperhidrosis: experience with pharmacological therapy using oxybutynin. Clinics. 2014;69:608-14.

5. Glaser DA. Oral medications. Dermatol Clin. 2014;32:527-32.

6. Glaser DA, Galperin TA. Managing hyperhidrosis: emerging therapies. Dermatol Clin. 2014;32:549-53.

7. Walling HW. Systemic therapy for primary hyperhidrosis: a retrospective study of 59 patients treated with glycopyrrolate or clonidine. J Am Acad Dermatol. 2012;66:387-92.

8. Baumgartner F. Invited commentary. Ann Thorac Surg. 2014;98:1797-803.

9. Nicolaou M, Paes T, Wakelin S. Blushing: an embarrassing condition, but treatable. Lancet. 2006;367:1297-8.

10. Drummond PD. A caution about surgical treatment for facial blushing. Br J Dermatol. 2000;142:194-5.

11. Adair A, George ML, Camprodon R, Broadfield JA, Rennie JA. Endoscopic sympathectomy in the treatment of facial blushing. Ann R Coll Engl. 2005;87: 358-60.

12. Jadresic E, Suarez C, Palacios E, Palacios F, Matus P. Evaluating the efficacy of endoscopic thoracic sympathectomy for generalized social anxiety disorder with blushing complaints: a comparison with sertraline and no treatment - Santiago de Chile 2003-2009. Innov Clin Neurosci. 2011;8:2.

13. Licht PB, Ladegaard L, Pilegaard HK. Thoracoscopic sympathectomy for isolated facial blushing. Ann Thorac Surg. 2006;81:1863-6.

Key Words: complications, hyperhidrosis, surgery, sympathectomy, sympathotomy 